\title{
Niedriglohnfalle Minijob
}

\author{
Minijobs prägen die Arbeitszeit- und Beschäftigungsmuster auf dem deutschen \\ Arbeitsmarkt. Mittlerweile ist hierzulande jedes fünfte Beschäftigungsverhältnis \\ ein Minijob. Der im europäischen Vergleich hohe Anteil dieser steuer- und abgaben- \\ rechtlich speziell regulierten Beschäftigungsform ist mit Folgekosten verbunden. \\ Sie entstehen auf individueller Ebene, weil Minijobs per se keine ausreichend \\ materielle und soziale Sicherung begründen. In Verbindung mit einer verbreiteten \\ gesetzeswidrigen Anwendung im Betrieb wird der Minijob in der Regel zur Niedrig- \\ lohnfalle, weshalb die Ausbreitung und Verfestigung des Niedriglohnsektors auf \\ dem deutschen Arbeitsmarkt nicht zuletzt auf den hohen Anteil von Minijobs zurück- \\ zuführen ist.
}

DOROTHEA VOSS, CLAUDIA WEINKOPF

\section{Einleitung}

Minijobs haben eine Sonderstellung im deutschen Beschäftigungssystem: Bis zur Einkommensgrenze von $400 €$ entrichten Beschäftigte keine Steuern und Abgaben an die sozialen Sicherungssysteme. Im Gegenzug erwerben sie im Minijob keine bzw. kaum eine eigenständige soziale Absicherung. Jedes fünfte Beschäftigungsverhältnis (20,8 \%) auf dem deutschen Arbeitsmarkt ist mittlerweile ein Minijob. Bei starkem Wachstum dieser Beschäftigungsform seit der Reform im Jahr 2003 verteilen sich die insgesamt knapp 7,4 Mio. Minijobs auf 4,93 Mio. ausschließlich und 2,45 Mio. im Nebenjob ausgeübte geringfügige Beschäftigungsverhältnisse (Angaben für Dezember 2010: Bundesagentur für Arbeit 2011).

In diesem Beitrag steht die Funktion von Minijobs in der Beschäftigungspolitik von Unternehmen im Vordergrund. Auf Basis empirischer Ergebnisse wird gezeigt, dass Minijobs in vielen Unternehmen die Funktion einer „ExitOption" haben, weil gesetzlich und kollektivvertraglich fixierte Standards für die Arbeitswelt unterlaufen werden. Dass der Minijob in der Regel zur Niedriglohnfalle geworden ist, zeigt sich darin, dass im Jahr 2009 fast $90 \%$ der geringfügig Beschäftigten Stundenlöhne unterhalb der Niedriglohnschwelle bezogen. Eine Analyse der Interessenkonstellationen auf betrieblicher Ebene veranschaulicht, dass es wenig Verbündete für eine wirksame Durchsetzung geltenden Rechts - also für die Gleichbehandlung von geringfügig Beschäftigten - gibt. Auch aus diesem Grund wird abschließend die These vertreten, dass die negativen Folgewirkungen für die meist weiblichen Beschäftigten in geringfügigen Beschäftigungsverhältnissen sowie die Absenkung von sozialen Standards auf dem gesamten Arbeitsmarkt am ehesten durch eine Beseitigung der steuer- und abgabenrechtlichen Sonderstellung von Minijobs verhindert werden können.

\section{Der besondere Status der geringfügigen Beschäftigung}

Das geringfügige Beschäftigungsverhältnis wurde mit dem Sozialgesetzbuch IV im Jahre 1971 eingeführt $^{\mathbf{0}}$ und sah schon damals die bis heute gültige und damit konstitutive Steuer- und Abgabenfreiheit bis zu einer bestimmten Einkommensgrenze vor. Obwohl es bereits in den 1980er und 90er Jahren eine Reihe von Gesetzentwürfen seitens der SPD gab, die in einer kritischen Perspektive eine Reform der geringfügigen Beschäftigung anmahnten - etwa

(1) Freigrenzen in der Sozialversicherung gab es allerdings bereits in der Weimarer Republik (Knospe 2007, S. 9). 
in dem Antrag zur „Verbesserung der Arbeits- und Lebensbedingungen von Frauen durch Abschaffung der geringfügigen Beschäftigung“" (Deutscher Bundestag 1989) oder im Gesetzentwurf „zur Beseitigung des Missbrauchs der Geringfügigkeitsgrenze in der Sozialversicherung" (Deutscher Bundestag 1994) - wurde die geringfügige Beschäftigung erst in der ersten Legislaturperiode der rot-grünen Bundesregierung im Jahre 1999 reformiert. Mit der Reform wurde angestrebt, die geringfügige Beschäftigung statistisch besser zu erfassen und in die Finanzierung der sozialen Sicherungssysteme teilweise mit einzubeziehen, ohne dass dadurch Belastungen für die ausschließlich geringfügig Beschäftigten entstehen sollten. Eine weitere Zielsetzung bestand darin, „die Ausweitung dieser Beschäftigung mittelfristig einzudämmen“ (Deutscher Bundestag 1999, S. 10).

Mit der neuerlichen Reform im Jahre 2003 wurde eine politische Kehrtwende vollzogen. Nun stand nicht mehr die Eindämmung der geringfügigen Beschäftigung auf der politischen Agenda, sondern in den nun als „Minijobs“ bezeichneten geringfügigen Beschäftigungsverhältnissen wurde die Chance gesehen, den Arbeitsmarkt zu flexibilisieren und Anreize für die Aufnahme einer Beschäftigung auch im unteren Einkommensbereich zu schaffen (Deutscher Bundestag 2003). Vor diesem Hintergrund wurden mehrere Änderungen beschlossen, die auf eine Ausweitung der Minijobs zielten: So wurde die Geringfügigkeitsgrenze von $325 €$ auf $400 €$ angehoben, die bis dahin geltende Arbeitszeitbegrenzung auf maximal 15 Wochenstunden ${ }^{2}$ wurde ersatzlos gestrichen und die erst 1999 eingeführte Steuerund Abgabenpflicht für geringfügige Nebenjobs wieder abgeschafft.

\subsection{Anreize für Beschäftigte und Unternehmen}

Für Beschäftigte besteht durch die Befreiung von Steuern und Sozialversicherungsabgaben ein monetärer Anreiz zur Aufnahme einer geringfügigen Beschäftigung. Allerdings müssen sich geringfügig Beschäftigte einen Minijob auch „leisten“ können, weil ein maximales Einkommen von $400 €$ im Minijob weder Existenz sichernd ist noch eigenständige Ansprüche in der gesetzlichen Kranken- und Pflegeversicherung, der Arbeitslosenversicherung und nur geringe Ansprüche in der gesetzlichen Rentenversicherung ${ }^{3}$ aufgebaut werden. Oftmals beziehen ausschließlich geringfügig Beschäftigte die soziale Sicherung über abgeleitete Ansprüche in der Ehe oder vom Sozialstaat, wenn aufstockende Leistungen beantragt werden (vgl. Bäcker/Neuffer sowie Dingeldey et al. in diesem Heft). Jedoch sind Minijobs auch in sogenannten prekär-diskontinuierlichen Erwerbsverläufen verbreitet, also bei denen, die sich eine Erwerbstätigkeit im Minijob materiell und sozial eigentlich nicht leisten können, aber keine Alternative auf dem Arbeitsmarkt vorfinden (vgl. Klenner/Schmidt in diesem Heft).

Vergleichbare monetäre Anreize wie für die Beschäftigten mit Minijobs sieht die gesetzliche Regulierung für Arbeitgeber nicht vor. Ihre Abgabenquote für Minijobs liegt seit Mitte 2006 bei etwa $30 \%$ des Monatsentgeltes und damit deutlich höher als für sozialversicherungspflichtige Beschäftigung (etwa $21 \%$ ). ${ }^{\oplus}$ Auch mit Blick auf arbeitsrechtliche und kollektivvertragliche Regelungen bietet die geringfügige Beschäftigung - de jure - den Arbeitgebern keine Vorteile, denn die in diesem Erwerbsstatus Beschäftigten sind entsprechend dem Benachteiligungsverbot von Teilzeitbeschäftigten der sozialversicherungspflichtigen Beschäftigung gleichgestellt und haben somit einen Anspruch darauf, bei der Entlohnung gegenüber sozialversicherungspflichtig Teilzeit- bzw. Vollzeitbeschäftigten nicht diskriminiert zu werden. ${ }^{\ominus}$ Hinsichtlich der Personal- bzw. Lohnnebenkosten ist daher zu konstatieren, dass die Regulierung der geringfügigen Beschäftigung eher negative Anreize für Arbeitgeber setzt, weshalb der starke Anstieg der geringfügigen Beschäftigung von rund 5,5 Mio. auf 7,4 Mio. im Zeitraum 20032010 aus Arbeitgebersicht auf den ersten Blick nicht erklärbar scheint.
(2) Seit der ersatzlosen Streichung der Arbeitszeitbegrenzung von 15 Wochenstunden im Minijob ist nun theoretisch möglich, dass ein Minijob bei sehr geringem Stundenlohn auch als Vollzeittätigkeit ausgeübt wird. Auswertungen mi dem Sozio-oekonomischen Panel (SOEP) zeigen, dass die vertragliche Arbeitszeit im Minijob im Jahr 2008 im Durchschnitt bei 12,8 Wochenstunden lag, weshalb der Minijob in der Regel als kurze Teilzeitbeschäftigung ausgeübt wird (Weinkopf 2011, S. 19).

3 Geringfügig Beschäftigte erwerben durch die Sozialversicherungsabgaben der Arbeitgeber geringe Ansprüche in der gesetzlichen Rentenversicherung (GRV). Es besteht zudem die Möglichkeit, die Rentenbeiträge im Minijob freiwillig auf den allgemeinen Rentenversicherungsbeitrag von 19,9\% aufzustocken, um höhere Rentenansprüche zu erwerben. Von dieser Möglichkeit machen jedoch nur rund 5\% der MinijobBeschäftigten Gebrauch (Minijobzentrale 2011, S. 12).
(4) Seit Juli 2006 entrichten Arbeitgeber in der geringfügigen Beschäftigung 15\% Abgaben an die gesetzliche Rentenversicherung (GRV), 13\% an die gesetzliche Krankenversicherung (GKV) und $2 \%$ pauschale Lohnsteuer. In der sozialversicherungspflichtigen Beschäftigung sind die entsprechenden Abgaben 9,95\% an die GRV, 7,3\% an die GKV, 1,5\% an die Arbeitslosenversicherung, 0,975\% an die Pflegeversicherung und $1,6 \%$ an die Unfallversicherung.

5 Der auf europäischer Ebene vereinbarte Grundsatz "pro rata temporis“ ist in der Richtlinie 97/81/EG des Europäischen Rates festgeschrieben. Diese Richtlinie ist durch das Teilzeit- und Befristungsgesetz, insbesondere das Verbot der Diskriminierung (TzBfG §4) in geltendes deutsches Recht umgesetzt worden. 


\subsection{Verzerrungen auf dem Arbeitsmarkt durch die Regulierung der geringfügigen Beschäftigung}

Der steuer- und abgabenrechtliche Sonderstatus von Minijobs führt in mehrfacher Hinsicht zu Verzerrungen auf dem Arbeitsmarkt: Erstens wird durch die Anreize für Beschäftigte, eine geringfügige Beschäftigung anzunehmen, die geschlechtsspezifische Verteilung des gesamtwirtschaftlichen Arbeitszeitvolumens beeinflusst. Der in Deutschland im europäischen Vergleich hohe Anteil von Frauen in kurzen Teilzeitbeschäftigungsverhältnissen wird durch die MinijobRegulierung geradezu künstlich aufrechterhalten und zementiert (vgl. Voss-Dahm 2011). ${ }^{\boldsymbol{0}}$ Nicht nur vor dem Hintergrund demografischer Entwicklungen ist kritisch zu hinterfragen, ob eine Regelung zeitgemäß ist, die dazu motiviert, das Arbeitsvermögen mehrheitlich qualifizierter Frauen quasi „stillzulegen“ - mit allen negativen Folgen für deren materielle und soziale Absicherung. Auch die Arbeitszeitlücke zwischen Männern und Frauen, die in Deutschland im Jahr 2009 gemessen in Vollzeitäquivalenten 21,5\% betrug, wird durch die Minijob-Regulierung konserviert (Sachverständigenkommission 2011, S. 92). Folgerichtig kommt die Sachverständigenkommission zur Erstellung des Ersten Gleichstellungsberichts der Bundesregierung (ebd. S. 135) zu dem Urteil, dass die „gegenwärtige Minijobstrategie [...] aus der Perspektive der Geschlechtergleichstellung [...] als desaströs“ zu bezeichnen ist. Zweitens kommt es zu Verzerrungen auf dem Arbeitsmarkt, weil es bei hohen Anteilen von Minijobs insbesondere in einigen Dienstleistungsbranchen gerade für diejenigen relativ schwerer wird, eine Beschäftigung zu finden, die aufgrund des Haushaltskontexts auf eine sozialversicherungspflichtige Beschäftigung angewiesen sind bzw. es ablehnen, ohne eigenständige soziale Sicherung bzw. nur für ein geringes Monatseinkommen erwerbstätig zu sein. Und drittens werden steuer- und abgabenpflichtige Überstunden gegenüber den im Minijob als Nebentätigkeit steuer- und abgabenfrei geleisteten Arbeitsstunden diskriminiert. Es ist also ökonomisch rational, Arbeitsstunden im Nebenjob statt Überstunden im Hauptjob zu leisten, was arbeitsmarkt- und sozialpolitisch schwerlich zu begründen ist (Bäcker 2007, S. 121)

\section{Der Minijob als Exit-Option in der betrieblichen Praxis}

Empirische Untersuchungen zeigen eine Diskrepanz zwischen der Regulierungsebene und ihrer Anwendung in der betrieblichen Praxis: Trotz des gesetzlich fixierten Verbots, (geringfügig) Teilzeitbeschäftigte mit Blick auf Entlohnung und gesetzlich verbriefte soziale Rechte zu diskriminieren, haben sich in der Unternehmenspraxis „Spielregeln“ - und in einem institutionalistischen Verständnis kann Regulie- rung durchaus als „Spielregel“ für Akteure in dem relevanten gesellschaftlichen Feld angesehen werden (vgl. North 1990) - in der Anwendung der Minijob-Regulierung herausgebildet, die von den gesetzlichen Vorgaben deutlich abweichen.

Solchen Diskrepanzen zwischen der Regulierungs- und Anwendungsebene wird in Studien jüngeren Datums vermehrt Aufmerksamkeit gewidmet. Artus (2010, S. 323) schlussfolgert auf Basis ihrer Untersuchungen zu industriellen Beziehungen in sogenannten peripheren Wirtschaftssegmenten, dass „Institutionenregeln und konkrete Akteurspraktiken $[\ldots]$ als nur bedingt verkoppelte Phänomene gedacht werden (müssten)“. In Untersuchungen über Formen des institutionellen Wandels wurde herausgearbeitet, dass regelwidriges Verhalten von Akteuren oftmals die Ursache für Veränderungen und Verschiebungen im Beschäftigungssystem ist (Streeck/Thelen 2005). Die Diskrepanz kann sich auch durch sogenannte Exit-Optionen vergrößern. Exit-Optionen sind de jure oder de facto existierende Ausnahmetatbestände bzw. Regelungslücken in einem ansonsten weitgehend regulierten Beschäftigungssystem (Bosch et al. 2010, S. 114ff.). In einem international vergleichenden Projekt zu niedrig entlohnten Tätigkeiten wurden Exit-Optionen insbesondere in den Ländern identifiziert, für die ein dichtes Netz aus gesetzlichen Regelungen und korporatistischer Interessenregulierung typisch ist (Gautié/Schmitt 2010). In Deutschland sind derartige Exit-Optionen durch Minijobs dieser Analyse zufolge gegeben. Exit-Optionen können von untergeordneter Bedeutung für das Gesamtsystem bleiben. Sofern sie aber auf eine starke Bedarfs- oder Interessenlage treffen - sei es, weil Unternehmen eine Möglichkeit sehen, Wettbewerbsdruck zu reduzieren, oder auch, weil Beschäftigte Abgaben sparen können-, können sie zu Auslösern kleinerer oder größerer Dammbrüche werden, die den eigentlich vorgesehenen institutionellen Rahmen brüchig machen und in deren Folge sich die Angebots- und Nachfrageverhältnisse auf dem Arbeitsmarkt nach anderen Regeln als den politisch intendierten abspielen (vgl. Sengenberger 1987, S. 268).

\subsection{Niedrige Entlohnung im Minijob}

Entsprechend des Benachteiligungsverbots von Teilzeitbeschäftigten haben geringfügig Beschäftigte Anspruch auf die gleichen Bruttostundenlöhne wie in einer vergleichbaren sozialversicherungspflichtigen Beschäftigung. Es gibt jedoch zahlreiche Hinweise darauf, dass hiervon in der Praxis abgewichen wird: Nach Auswertungen des Statistischen Bundesamtes verdienten geringfügig beschäftigte

\footnotetext{
(6 Im Vergleich von 19 europäischen Ländern war im Jahr 2008 nur in den Niederlanden der Anteil der Frauen, die weniger als 20 Wochenstunden erwerbstätig waren, mit $31,2 \%$ höher als in Deutschland mit 20,8\% aller Frauen in diesem Arbeitszeitintervall (Lehndorff et al. 2010, S. 21 ff.).
} 
ABB. 1

\section{Abhängig Beschäftigte nach Stundenlohnstufen*}

- unter $5 € \quad 5,00 €$ bis $5,99 € \quad 6,00$ bis $6,99 € \square 7,00$ bis $7,99 € \quad 8,00$ bis $8,49 €$
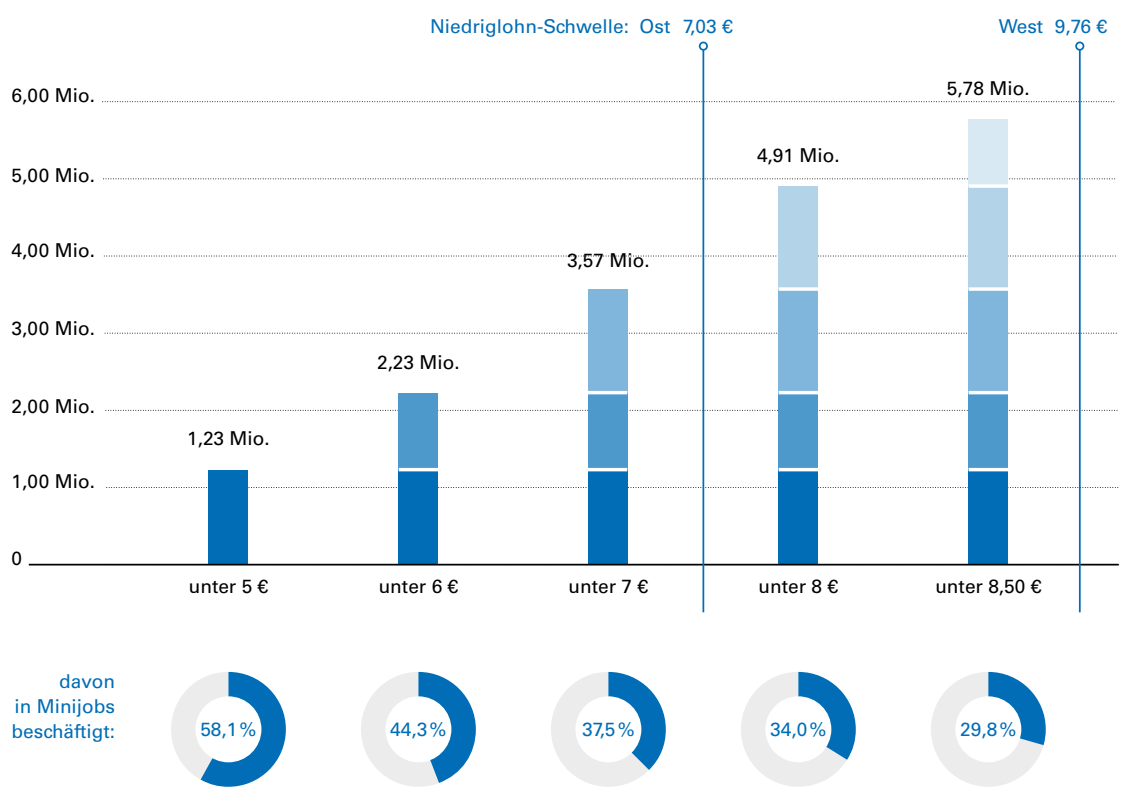

* Hauptbeschäftigung, ohne Schüler, Studierende, Rentner und Nebenjobs.
Milteilungern schäftigten. Betrachtet man allerdings nur die Beschäftigten in Minijobs, dann ist festzustellen, dass mehr als drei Viertel $(76,4 \%)$ weniger als $8,50 €$ pro Stunde verdienen.

Derart deutliche Befunde von Lohndifferenzen zwischen geringfügig und sozialversicherungspflichtig Beschäftigten verlangen insbesondere deshalb eine Erklärung, weil sie weder auf die Regulierung der geringfügigen Beschäftigung selbst noch auf die Qualifikationsprofile der Beschäftigten in Minijobs zurückzuführen sind.

Es ist vielmehr die Anwendungspraxis, die den Minijob zur Niedriglohnfalle macht. Dies kann anhand eines Beispiels verdeutlicht werden (Abbildung 2): Eine sozialversicherungspflichtig Beschäftigte erhält für eine bestimmte Tätigkeit einen Bruttostundenlohn von 13,50 €. Ist sie verheiratet, kinderlos und wird mit Lohnsteuerklasse V veranlagt, erhält sie einen Nettostundenlohn von rund $7 €$ (gerechnet mit www.nettolohn.de). Eine geringfügig Beschäftigte würde für die gleiche Tätigkeit bei gesetzeskonformer Umsetzung der Minijob-Regelung ebenso 13,50€ pro Stunde erhalten, die dann steuer- und abgabenfrei bliebe (brutto = netto). In der betrieblichen Praxis wird der Stundenlohn der geringfügig Beschäftigten aber häufig mit einem Lohnabschlag belegt. Sie erhält beispielsweise nur $7 €$ und damit den Stundenlohn, den sie netto erhalten würde, wenn sie die Tätigkeit als sozialversicherungspflichtig Beschäftigte ausgeübt hätte. Der Arbeitgeber zahlt eine pauschale Abgabe von $30 \%$ auf den Stundenlohn von $7 €$ und spart damit Personalkosten gegenüber den Lohnkosten für eine sozialversicherungspflichtige Beschäftigung.

Aus empirischen Analysen wissen wir, dass solche Praktiken in der Arbeitswelt keinesfalls Ausnahmen sind. So sehen beispielsweise in einem großen tarifgebundenen Unternehmen des Lebensmitteleinzelhandels mündliche Absprachen zwischen Geschäftsführung und Betriebsrat nach Tätigkeit gestaffelte Lohnhöhen innerhalb der geringfügigen Beschäftigung vor. Neben dem Tarifgitter für die sozialversicherungspflichtig Beschäftigten existiert also ein zweites, informelles Tarifgitter für geringfügig Beschäftigte, deren Anteil an der Belegschaft in den untersuchten Filialen $35 \%$ beträgt. Zum Untersuchungszeitpunkt im Jahre 2006 erhielten Schülerinnen und Schüler einen Stundenlohn von $5,15 €$, was gegenüber der damals geltenden tariflichen Entgeltgruppe einen Lohnabschlag von $26 \%$ bedeutete. Erwachsene geringfügig Beschäftigte, die in der Warenverräumung tätig waren, erhielten einen Stundenlohn von $6,80 €$ (29\% Lohnabschlag) und geringfügig Beschäftigte im Kassen- und Bedienbereich einen Stundenlohn von $8,00 €$, was einem Abschlag von $33 \%$ gegenüber der entsprechenden tariflichen Gehaltsgruppe entsprach (vgl. Voss-Dahm 2009,

Geringfügig beschäftigte Frauen verdienten durchschnittlich 9,07 €; Männer 8,78 €. In Westdeutschland lag der durchschnittliche Stundenlohn mit 9,20€ deutlich höher als in Ostdeutschland und Berlin (7,16 €) (Wingerter 2009, S. 1088). 
S. 233ff.). Zu ähnlichen Ergebnissen kam auch eine Studie im sächsischen Einzelhandel, nach der geringfügig Beschäftigte zum Teil nur die Hälfte des Tariflohns erhielten (vgl. Benkhoff/Hermet 2008). Ebenso wird von systematischen Entlohnungsunterschieden von geringfügig und sozialversicherungspflichtigen Beschäftigten beim Textildiscounter KIK und dem Lebensmitteldiscounter Netto berichtet (vgl. Hinz in diesem Heft).

Auch in der Gesundheits- und Sozialbranche werden geringfügig Beschäftigte offensichtlich nicht regelungskonform entlohnt. Bisher sieht der Reformtarifvertrag des Deutschen Roten Kreuzes in einer gesonderten Anlage spezielle Lohnhöhen für geringfügig Beschäftigte vor. Diese liegen zwischen 6,75€ für Tätigkeiten, die nur geringe Fachkenntnis erfordern, und 8,50€ für Tätigkeiten, die mit abgeschlossener Ausbildung ausgeübt werden. Die Gewerkschaft ver.di hat diese Anlage zum 31. Oktober 2011 gekündigt und erwartet, dass diese gesetzeswidrige Regelung ohne Nachwirkung ersatzlos gestrichen wird. Die Klage einer Pflegehilfskraft verweist darauf, dass auch bei der Caritas als einem weiteren großen Arbeitgeber der Branche sozialversicherungspflichtig und geringfügig Beschäftigte systematisch unterschiedlich entlohnt werden. Die betreffende Pflegehilfskraft wurde mit dem gesetzlichen Mindestlohn der Pflegebranche in Höhe von 8,50 € entlohnt, was einen Lohnabschlag von $25 \%$ gegenüber der tariflichen Entlohnung von sozialversicherungspflichtig Beschäftigten in gleicher Tätigkeit bedeutet. ${ }^{8}$

Die Beispiele zeigen die Diskrepanz zwischen Regelungsund Anwendungsebene in der geringfügigen Beschäftigung. Für die Beschäftigten erweist sich die Annahme, in Minijobs werde „brutto gleich netto" und damit mehr als in einer sozialversicherungspflichtigen Beschäftigung verdient, damit faktisch oft als Illusion. Durch Lohnabschläge kommt der „Vorteil“ durch die Steuer- und Abgabenbefreiung nicht wie politisch intendiert den Beschäftigten im Minijob, sondern den Unternehmen zugute, die die höhere Abgabenquote von $30 \%$ für Minijobs (gegenüber etwa $21 \%$ in der sozialversicherungspflichtigen Beschäftigung) durch erheblich niedrigere Stundenlöhne kompensieren. Durch diese betriebliche Praxis wird der Minijob zu einer Exit-Option für Unternehmen, die ihnen faktisch eine Reduktion der Personalkosten ermöglicht.

\subsection{Unterlaufen arbeitsrechtlicher und kollektivvertraglicher Standards}

Das im Teilzeit- und Befristungsgesetz enthaltene Diskriminierungsverbot der Teilzeitbeschäftigung wird nicht nur mit Blick auf das Entgelt, sondern auch bezogen auf andere gesetzlich verbriefte soziale Ansprüche verletzt. Bezogen auf die Lohnfortzahlung im Krankheitsfall führt Winkel (2005) aus, dass die zur Verfügung stehenden Gelder bei der Minijobzentrale zu einem wesentlich geringeren prozentualen Anteil abgerufen werden als für sozialversicherungspflichtig Beschäftigte. Das kann als ein Indiz dafür
ABB. 2

\section{Niedriglohnfalle Minijob}

Beispielrechnung

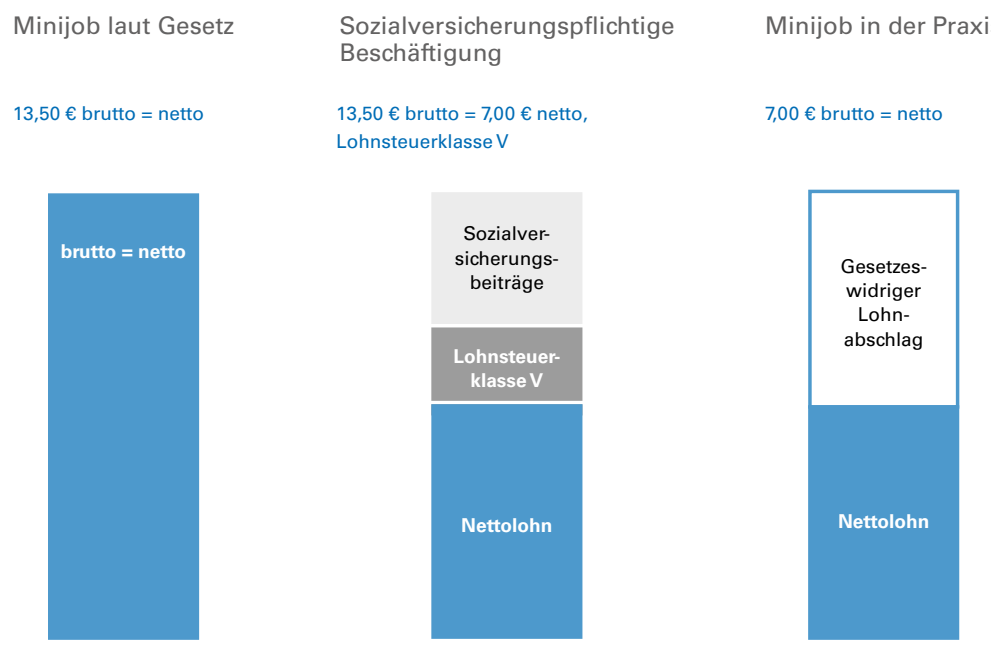

Quelle: Darstellung der Autorinnen.

gewertet werden, dass die Lohnfortzahlung im Krankheitsfall im Minijob weniger in Anspruch genommen wird. Untersuchungen im Einzelhandel zeigen Abstufungen: Die nach dem Manteltarifvertrag tarifvertraglich vereinbarte Lohnfortzahlung von zehn Wochen gilt in einem der größten filialisierten Unternehmen des Lebensmitteleinzelhandels nicht für Beschäftigte im Minijob, denen im Krankheitsfall nur die gesetzliche Lohnfortzahlung von sechs Wochen gewährt wird (Voss-Dahm 2009, S. 237).

Vor dem Hintergrund einer in Unternehmen und häufig auch bei Beschäftigten verbreiteten Meinung, der Minijob sei eben nur „Mini“ und damit eine abgespeckte Form des normalen Beschäftigungsverhältnisses, verwundern Hinweise auf ein Unterlaufen weiterer arbeits- und kollektivvertraglicher Standards nicht: In einer Studie im Reinigungsgewerbe sind Gather et al. (2005, S. 142ff.) auf eine Reihe von Abweichungen gestoßen, die Zuschläge und Jahressonderzahlungen betrafen. Auch von der Verpflichtung, Arbeitsstunden nachzuarbeiten, die aufgrund gesetzlicher Feiertage nicht geleistet wurden, wurde berichtet. Darüber hinaus wurden Überstunden nicht (voll) vergütet oder Leitungsaufgaben bei der Eingruppierung nicht berücksichtigt. Die Studie im sächsischen Einzelhandel hat gezeigt, dass geringfügig Beschäftigte üblicherweise kein Urlaubsgeld erhalten, kaum in Weiterbildung einbezogen sind und

8 Vgl. Urteil (AZ:18 Sa 2049/10) des Landesarbeitsgerichts Hamm am 29.07.2011. 
im Wesentlichen auf Abruf arbeiten bzw. nach kurzen $\mathrm{Ar}$ beitseinsätzen vorzeitig auch wieder nach Hause geschickt werden (Benkhoff/Hermet 2008). Dass gesetzlich verbriefte soziale Ansprüche im Minijob nicht gewährt werden, deutet auch der Sachverständigenrat zur Begutachtung der gesamtwirtschaftlichen Entwicklung (2009, Ziffer 553) an. Als mögliche Gründe nennt er unter anderem die „Unkenntnis der Arbeitnehmer über ihre Rechte oder dass diese wegen der geringeren Bedeutung des Beschäftigungsverhältnisses und der Tätigkeiten nicht auf ihren vollen Rechten insistieren“.

\subsection{Gibt es Akteure für eine Durchsetzung des Gleichbehandlungsgebots im Betrieb?}

Vor dem Hintergrund dieser Befunde stellt sich die Frage, weshalb derartige Verstöße gegen geltendes Recht im Betrieb offensichtlich mehr oder weniger stillschweigend toleriert werden. Ebenso ist zu fragen, welche Arbeitsmarktakteure an einem Aufbrechen dieser Situation ein Interesse haben könnten. Denn immerhin wäre zu vermuten, dass die durch die Exit-Option Minijob in Gang gesetzten Abwärtsspiralen mehr Verlierer als Gewinner hervorbringen, was dafür spräche, dass sich Widerstand formieren würde. Die folgenden Ausführungen zeigen jedoch, dass dies nicht der Fall ist.

Für Unternehmen ist die Exit-Option Minijob ein Schlupfloch zur Senkung von Personalkosten. In drei privaten Dienstleistungsbranchen, nämlich dem Einzelhandel, dem Gastgewerbe und dem Reinigungsgewerbe, mit besonders hohen Anteilen der geringfügigen Beschäftigung dürfte diese Praxis besonders verbreitet sein (vgl. Hinz; Rothe et al. sowie Riedel in diesem Heft). Rund 5,7 Mio. Beschäftigungsverhältnisse werden für diese drei Branchen ausgewiesen; der Anteil der geringfügigen Beschäftigung liegt zusammengenommen bei $40 \%{ }^{\boldsymbol{\bullet}}$ Die Branchen zeichnen sich dadurch aus, dass der Frauen- und Teilzeitanteil traditionell hoch ist, die Erstellung der Dienstleistungen arbeitsintensiv ist und Schwankungen des Arbeitsanfalls sowie Arbeitseinsätze am Morgen, am Abend und am Wochenende arbeitsorganisatorisch zu bewältigen sind. Hinzu kommt, dass die Konkurrenz der Unternehmen durch Kostenwettbewerb geprägt wird, mittels Kostensenkungen Marktanteile also gehalten bzw. erhöht werden können. Gehört die Exit-Option Minijob in einem solchen Umfeld einmal zum Set an „Spielregeln“ in der Branche, ist der Minijob hier nicht nur eine Beschäftigungsform, die der Erhöhung der betrieblichen Einsatzflexibilität und dem Abfangen von Spitzen des Arbeitsanfalls dient, sondern auch eine Senkung der Personalkosten ermöglicht. Unternehmen, die sich im Wettbewerb über niedrige Kosten positionieren, werden daher kein Interesse an einer Veränderung der gegenwärtigen Beschäftigungspraxis haben. Andere Unternehmen äußern durchaus Kritik an dem Sog auf die „low road“, weil Standards in der Beschäftigung und in der Dienstleistungsqualität unter Druck geraten. Sich als einzelnes Unternehmen für eine regelkonforme Entlohnung in der geringfügigen Beschäftigung zu entscheiden, dürfte vom Markt jedoch kaum belohnt werden und erscheint als riskante Strategie.

Die geringfügig Beschäftigten selbst wiederum sind keine homogene Erwerbsgruppe und je nach Haushaltskontext und Dauer des geringfügigen Beschäftigungsverhältnisses in der Erwerbsbiografie von den Konsequenzen unterschiedlich betroffen (vgl. Bäcker/Neuffer in diesem Heft). Selbst wenn geringfügig Beschäftigte über ihre Rechte und die ihnen zustehenden Leistungen im Minijob informiert sind, spricht einiges dafür, dass sie aufgrund ihres marginalisierten Status innerhalb der Belegschaften keine starke Machtposition für die Durchsetzung der ihnen zustehenden Leistungen haben. Für geringfügig Beschäftigte im Nebenjob sowie ausschließlich geringfügig Beschäftigte, die die soziale und materielle Sicherung über den Haushaltskontext beziehen, hat der Minijob den Status eines - wenn auch häufig notwendigen - Zuverdienstes. Selbst wenn sie die Praxis als „ungerecht“ empfinden, ist allerdings zu bezweifeln, dass sie sich als Beschäftige mit (in der Regel) geringer Wochenarbeitszeit und daher kurzer Anwesenheit im Betrieb aktiv für eine gesetzeskonforme Umsetzung der Vorschriften im Bereich der geringfügig Beschäftigten einsetzen. Für geringfügig Beschäftigte, die auf eine „ertragreichere“ Beschäftigung als den Minijob angewiesen sind, aber keine Alternativen zum Minijob vorfinden, ${ }^{\circledR}$ verschlechtern sich die Beschäftigungsbedingungen durch das Vorenthalten kollektiv- und arbeitsrechtlicher Leistungen im Minijob spürbar. Es liegen keine Erkenntnisse über die Mobilisierungsbereitschaft dieser Gruppe innerhalb der geringfügigen Beschäftigung vor. Hinweise darauf, dass sie statt der VoiceOption eher die Exit-Option aus dem Minijob wählen würden, geben jedoch die Analysen von Klenner/Schmidt (in diesem Heft): Frauen mit diskontinuierlich-prekären Erwerbsverläufen präferieren klar eine Ausweitung ihrer Arbeitszeit, arbeiten deshalb unfreiwillig im Minijob und wechseln nach Möglichkeit in eine sozialversicherungspflichtige Beschäftigung (vgl. auch Wanger 2011).

Auch sozialversicherungspflichtig Beschäftigte dürften nur in begrenztem Maße als Verbündete für eine Veränderung der gegenwärtig praktizierten Exit-Option Minijob infrage kommen. Würden die Regelungen zur geringfügigen Beschäftigung im Betrieb gesetzeskonform umgesetzt,

( Für Dezember 2010 werden in der Beschäftigungsstatistik der Bundesagentur für Arbeit für den Einzelhandel rund 3,2 Mio. Beschäftigungsverhältnisse mit einem MinijobAnteil von $32 \%$ und für das Gastgewerbe 1,6 Mio. Beschäftigungsverhältnisse bei einem Minijob-Anteil von $49 \%$ ausgewiesen (Bundesagentur für Arbeit 2011). Riedel (in diesem Heft) beziffert die Anzahl der Beschäftigten in der Gebäudereinigung auf knapp 874.000 und den Anteil der geringfügigen Beschäftigung auf $53 \%$.

(1) So ist es etwa in der Gebäudereinigung angesichts des hohen Anteils von Minijobs außerhalb von Anleitungsfunktionen ausgesprochen schwierig, überhaupt ein sozialversicherungspflichtiges Beschäftigungsverhältnis zu erhalten. 
bekämen die Beschäftigten mit besonders kurzer Arbeitszeit netto mehr „heraus“ als sie selbst, die mehr Zeit im Betrieb verbringen und oft (aber nicht immer!) mehr Verantwortung für die betrieblichen Abläufe übernehmen. Ebenso schafft die Exit-Option Minijob bei hohem Kostendruck im Betrieb im ersten Moment Entlastung für sie selbst: Personalkosten werden bei den geringfügig Beschäftigten gespart und schützen damit zumindest eine Zeitlang vor Eingriffen im „eigenen Lager“. Wenn Minijobs aus Kostengründen ausgeweitet und damit teurere Arbeitsstunden durch billigere ersetzt werden, kann dies jedoch dauerhaft wieder negativ auf die sozialversicherungspflichtige Beschäftigung zurückschlagen.

Und wie ist die Haltung der Betriebsräte? Zunächst einmal ist für die drei genannten Branchen festzuhalten, dass die arbeitsplatznahe Absicherung der Beschäftigungsbedingungen eher die Ausnahme ist: Im Jahr 2009 gab es nur in etwa jedem zehnten Handelsbetrieb einen Betriebsrat und im Gastgewerbe bzw. in sonstigen Dienstleistungen lag der Anteil mit rund $3 \%$ noch deutlich niedriger (Ellguth/Kohaut 2010, S. 208). ${ }^{\oplus}$ Der Normalfall ist also, dass keine betriebliche Interessenvertretung auf die regelkonforme Umsetzung der Vorschriften für die geringfügige Beschäftigung achten kann. Und selbst wenn betriebliche Interessenvertretungen existieren, weisen Untersuchungen im Einzelhandel auf unterschiedliche Positionen hin (vgl. Voss-Dahm 2009; Hinz in diesem Heft): Manche Betriebsräte sehen keinen Spielraum, sich gegen den Kostendruck im Unternehmen zu stemmen, und akzeptieren die Ungleichbehandlung von geringfügig Beschäftigten als kleineres Übel gegenüber Eingriffen im Bereich der sozialversicherungspflichtig Beschäftigten. Andere sehen die Gefahr der Verdrängung von sozialversicherungspflichtiger Beschäftigung durch Minijobs und argumentieren strategisch wie diese Betriebsrätin: „Wenn wir nicht darauf achten, dass auch Minijobber tariflich entlohnt werden, dann fressen die uns nach und nach auf" (Voss-Dahm 2009, S. 235).

Zusammengefasst ist zu konstatieren, dass von einer breiten Unterstützerfront für eine gesetzeskonforme Anwendung der Vorschriften zur geringfügigen Beschäftigung im Betrieb (noch) keine Rede sein kann. Dies spricht dafür, dass entsprechende Reformvorschläge Überlegungen zur Mobilisierung und Durchsetzung des Gleichbehandlungsgrundsatzes mit einbeziehen müssten (vgl. Weinkopf 2011).

\section{Fazit: Niedriglohnfalle Minijob}

Acht Jahre nach der Reform der geringfügigen Beschäftigung fällt die Bilanz ihrer Wirkungen auf dem Arbeitsmarkt ernüchternd aus: Bei deutlicher Zunahme von Minijobs ist diese Beschäftigungsform mit Sonderstatus zum Einfallstor für betriebliche Verstöße gegen elementare Arbeitnehmerrechte geworden, weil weder wirksame Kontrollen zur Ein- haltung noch spürbare Sanktionen bei Verstößen gegen das Diskriminierungsverbot von Beschäftigungsverhältnissen aufgrund unterschiedlicher Arbeitszeitdauer existieren. Beschäftigte werden mit dem Trugschluss, sie machten mit einem Minijob ein gutes Geschäft, dazu verleitet, nur eine kurze Teilzeitbeschäftigung ohne eigenständige soziale Sicherung auszuüben. Bei genauerer Betrachtung können sich allerdings nur Erwerbspersonen diese Beschäftigungsform „leisten“, bei denen der Haushaltskontext oder der Sozialstaat früher oder später als Lückenbüßer ,,in die Bresche springen“. Minijobs werden zu zwei Dritteln von Frauen ausgeübt. Daher nähren geringfügige Beschäftigungsverhältnisse maßgeblich die für Deutschland typische geschlechtsspezifische Arbeitszeitlücke, die sich auch in Entgeltunterschieden zwischen Frauen und Männern niederschlägt. Der gesetzlich fixierte Sonderstatus Minijob ist daher faktisch ein institutionelles Hindernis auf dem Weg zur Gleichverteilung von Erwerbschancen von Frauen und Männern und blockiert - offensichtlich mit politischem Einverständnis - den notwendigen Ausbau der eigenständigen Sicherung, vor allem der Alterssicherung von Frauen. Jedwede Form von „Lockmittel“ in die kurze Teilzeit steht dem Ziel einer gerechteren Verteilung von Erwerbschancen auf dem Arbeitsmarkt daher diametral entgegen und erweist sich gesellschaftspolitisch als Sackgasse.

Die verbreitete Anwendungspraxis von Minijobs hat weitere Auswirkungen auf individueller Ebene und das gesamte Beschäftigungssystem: Wird die geringfügige Beschäftigung (rechtswidrig) als Exit-Option von tariflichen und gesetzlichen Bestimmungen genutzt, werden die betreffenden Beschäftigten benachteiligt und in der Regel mit Niedriglöhnen abgespeist. Gesetzeswidrige Lohnabschläge sowie das Vorenthalten von weiteren gesetzlichen und kollektivvertraglichen Ansprüchen haben nicht nur ein ZweiKlassensystem auf dem Arbeitsmarkt geschaffen und zu Spaltungen von Belegschaften geführt. Angesichts der quantitativen Bedeutung der geringfügigen Beschäftigung verschieben sich durch solche Praktiken auch soziale Standards im Sinne selbstverständlich geltender und geteilter Normen im gesamten Beschäftigungssystem nach unten. Unter Berücksichtigung aller genannter Fehlentwicklungen kann die Schlussfolgerung daher nur lauten: Abschaffung des Sonderstatus Minijob.
(1) Die Angaben beziehen sich auf privatwirtschaftliche Betriebe mit mindestens fünf Beschäftigten. 


\section{LITERATUR}

Artus, I. (2010): Interessenhandeln jenseits der Norm: Ein deutsch-französische Vergleich betrieblicher Interessenvertretung in peripheren und prekären Wirtschaftssegmenten, in: Industrielle Beziehungen 17 (4), S. 317-344

Bäcker, G. (2007): Was heißt hier "geringfügig“? Minijobs als wachsendes Segment prekärer Beschäftigung, in: Keller, B./Seifert, H. (Hrsg.): Atypische Beschäftigung - Flexibilisierung und soziale Risiken, Berlin, S. 107-126 Benkhoff, B./Hermet, V. (2008): Zur Verbreitung und Ausgestaltung geringfügiger Beschäftigung im Einzelhandel. Eine explorative Studie aus der Perspektive von Management und Beschäftigten, in: Industrielle Beziehungen 15 (1), S. 5-31

Bosch, G./Mayhew, K./Gautié, J. (2010): Industrial Relations, Legal Regulations, and Wage Setting, in: Gautié, J./Schmitt, J. (Hrsg.): Low Wage Work (a. a. O.) Bundesagentur für Arbeit (2011): Arbeitsmarkt in Zahlen. Beschäftigungsstatistik, Nürnberg, http://statistik.arbeitsagentur.de

Deutscher Bundestag (1989): Antrag der SPD-Fraktion. Verbesserung der Arbeits- und Lebensbedingungen von Frauen durch Abschaffung der gering fügigen Beschäftigung, Bundestagsdrucksache 11/5689 vom 15. November Deutscher Bundestag (1994): Gesetzentwurf der Fraktion der SPD. Entwurf eines Gesetzes zur Beseitigung des Mißbrauchs der Geringfügigkeitsgrenze in der Sozialversicherung. Bundestagsdrucksache 12/7108 vom 16. März Deutscher Bundestag (1999): Gesetzentwurf der Fraktionen SPD und BÜNDNIS 90/DIE GRÜNEN. Entwurf eines Gesetzes zur Neuregelung der geringfügigen Beschäftigung, Bundestagsdrucksache 14/280 vom 19. Januar 1999 Deutscher Bundestag (2003): Bericht der Bundesregierung zu den Auswirklungen des Gesetzes zur Neuregelung der geringfügigen Beschäftigungsver hältnisse auf den Arbeitsmarkt, die Sozialversicherung und die öffentlichen Finanzen, Drucksache 15/758 vom 31. März

Ellguth, P./Kohaut, S. (2010): Tarifbindung und betriebliche Interessenvertretung: Aktuelle Ergebnisse aus dem IAB-Betriebspanel 2009, in: WSI-Mitteilungen 63 (4), S. 204-209, http://www.boeckler.de/wsi_25394_25400.htm

Gather, C./Gerhard, U./Schroth, H./Schürmann, L. (2005): Vergeben und vergessen? Gebäudereinigung im Spannungsfeld zwischen kommunalen Diensten und Privatisierung, Hamburg

Gautié, J./Schmitt, J. (Hrsg.) (2010): Low Wage Work in the Wealthy World, New York

Knospe, A. (2007): Die Attraktivität der geringfügigen Beschäftigung im zeitlichen Wandel politisch motivierter Reformen, in: Die Sozialgerichtsbarkeit Zeitschrift für das aktuelle Arbeitsrecht 54 (1), S. 8-16

Lehndorff, St./Wagner, A./Franz, Ch. (2010): Arbeitszeitentwicklung in Europa, hrsg. von Th. Händel u. A. Troost, Fraktion der Vereinigten Europäischen Linken, Nordisch Grüne Linke - GUE/NGL, Brüssel

Minijobzentrale (2011): Aktuelle Entwicklungen im Bereich der geringfügigen Beschäftigung. 1. Quartal 2011, Essen

North, D. (1990): Institutions, Institutional Change and Economic Performance, Cambridge

Sachverständigenkommission zur Erstellung des Ersten Gleichstellungsberichtes der Bundesregierung (2011): Neue Wege - Gleiche Chancen. Gleichstellung von Frauen und Männern im Lebensverlauf. Gutachten der Sachverständigenkommission an das Bundesministerium für Familie, Senioren, Frauen und Jugend für den Ersten Gleichstellungsbericht der Bundesregierung, Essen/München
Sachverständigenrat (2009): Strategien für einen weiteren Beschäftigungsaufbau: Was nun getan werden muss (Ziffern 531 bis 600), Auszug aus dem Jahresgutachten 2008/2009, Wiesbaden, S. 311-343

Sengenberger, W. (1987): Arbeitsmarktsegmentation und Macht, in: Buttler, F./ Gerlach, K./Schmiede, R. (Hrsg.): Arbeitsmarkt und Beschäftigung. Neuere Bei träge zur institutionalistischen Arbeitsmarktanalyse, Frankfurt a. M./New York, S. $117-122$

Streeck, W./Thelen, K. (2005): Beyond Continuity - Institutional Change in Advanced Political Economis, New York

Voss-Dahm, D. (2009): Über die Stabilität sozialer Ungleichheit im Betrieb: Verkaufsarbeit im Einzelhandel, Berlin

Voss-Dahm, D. (2011): Erwerbsverläufe von Frauen im Einzelhandel. Gründe für die Entstehung geschlechtsspezifischer Ungleichheit im Betrieb, in: Klammer, U./Motz, M. (Hrsg.): Neue Wege - gleiche Chancen, Wiesbaden, S. $313-330$

Wanger, S. (2011): Viele Frauen würden gerne länger arbeiten, Institut für Arbeitsmarkt- und Berufsforschung, IAB-Kurzbericht (9), Nürnberg

Weinkopf, C. (2011): Minijobs - politisch-strategische Handlungsoptionen. Expertise im Rahmen des Projektes „Gesellschaftliche Wertschätzung von Dienstleistungen steigern! Dienstleistungsqualität - Arbeitsqualität - Zeitinnovationen", Berlin

Wingerter, Ch. (2009): Der Wandel der Erwerbsformen und seine Bedeutung für die Einkommenssituation Erwerbstätiger, in: Wirtschaft und Statistik (11), S. $1080-1098$

Winkel, R. (2005): Minijob-Bilanz: Kaum Lohnfortzahlung bei Krankheit und Mutterschaft, in: Soziale Sicherheit 53 (9), S. 292-298

Wolf, E. (2010): Lohndifferenziale zwischen Vollzeit- und Teilzeitbeschäftigten in Ost- und Westdeutschland, WSI-Diskussionspapier (174), Düsseldorf

\section{AUTORINNEN}

DOROTHEA VOSS, Dr., ist Referatsleiterin in der Abteilung Forschungsförderung der Hans-Böckler-Stiftung. Arbeitsschwerpunkte: Soziale Sicherung in Erwerbsarbeit; Wechselwirkungen von Beschäftigungspolitik in Unternehmen und institutionellen Rahmenbedingungen.

dorothea-voss@boeckler.de

CLAUDIA WEINKOPF, Dr., ist Stellvertretende Geschäftsführende Direktorin des Instituts Arbeit und Qualifikation (IAQ), Universität Duisburg-Essen. Arbeitsschwerpunkte: Arbeitsmarkt und Beschäftigung, Niedrig- und Mindestlöhne, Gender.

claudia.weinkopf@uni-due.de 